\title{
Characterization of Homogeneous and Heterogeneous Amyloid- $\beta 42$ Oligomer Preparations with Biochemical Methods and Infrared Spectroscopy Reveals a Correlation between Infrared Spectrum and Oligomer Size
}

Faraz Vosough and Andreas Barth*

Department of Biochemistry and Biophysics, Stockholm University, Stockholm, Sweden.

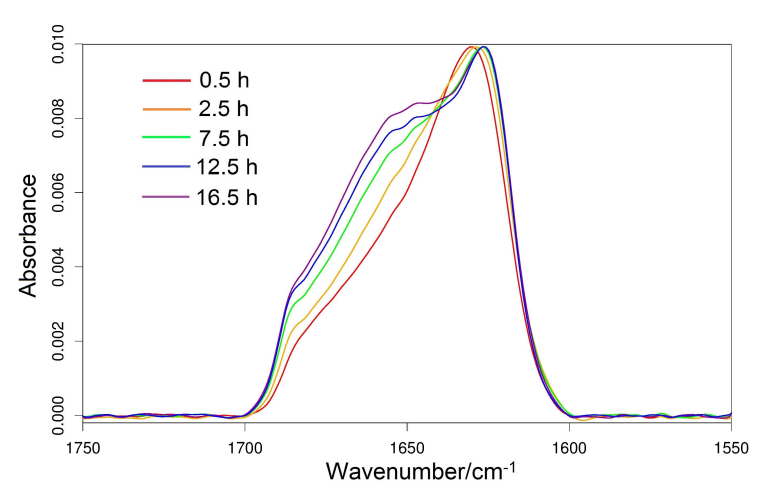

Figure S1. Infrared absorbance spectra after subtraction of a solvent spectrum. The spectra correspond to the second derivative spectra for the kinetic experiment shown in Figure 10 a. The red spectrum was recorded after 30 minutes at $0^{\circ} \mathrm{C}$. The other spectra were recorded at $37^{\circ} \mathrm{C}$. The spectra are normalized to a maximum absorbance of 0.01 . The original maximun absorbance for each spectrum was as follows: red ( $0.5 \mathrm{~h})$ : 0.011; orange ( $2.5 \mathrm{~h})$ : 0.013 ; green (7.5 h): 0.011 ; blue (12.5 h): 0.010; purple (16.5 h): 0.011 .

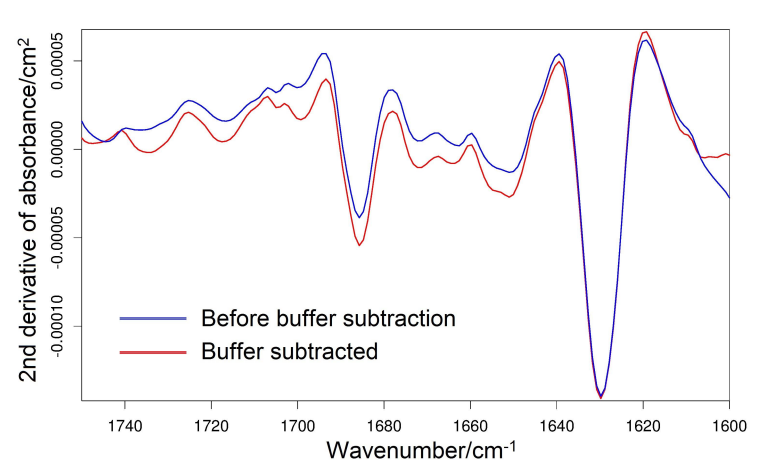

Figure S2. The effect of buffer subtraction on the second derivative of the infrared absorbance spectrum for $0.05 \%$ SDS-stabilized synthetic $A \beta 42$ oligomers (globulomers). Blue: before buffer subtraction, Red: buffer-subtracted. The comparison shows that the second derivative spectrum is only very little affected by solvent absorption. 